\title{
Erratum
}

\section{Spectra of Compact Locally Symmetric Manifolds of Negative Curvature}

\author{
J.J. Duistermaat, J.A.C. Kolk, and V.S. Varadarajan
}

Inventiones math. 52, 27-93 (1979)

In Remark 5.9, Case $\alpha$ real, read:

(as $\theta \alpha=-\alpha, X_{\alpha} \pm \theta X_{\alpha} \neq 0$ ).

In the proof of Proposition 5.16, read:

... since $A^{(\gamma)} \backslash A \cong \Gamma_{\gamma} \backslash M_{\gamma_{1}}, A / M_{\gamma_{1}}$ is compact, ...

In formula (9.3), read $\Gamma\left(\frac{n}{2}+1\right)^{-1}$ instead of $\Gamma\left(\frac{n}{2}+1\right)$

The conclusion on p. 89 , line 4 from below, is incorrect as it is based on the erronous assumption that Bérard's estimate (61) is obtained from his estimate (60) by integration. Instead, Bérard obtained his estimate (61) from a separate analysis of the integrals $I_{A}^{k, y}(\mu, x)$, integrated over $\mu$ from $-v$ to $v$, as he kindly pointed out in a letter to us.

In formula (9.5) the number of the formula is missing. Also the last term should have a minus sign.

In formula (9.6) read: $\|H\| \leqq \varepsilon^{-1}$. 